\title{
The European Federation of Organisations for Medical Physics Policy Statement No 14: The role of the Medical Physicist in the management of safety within the magnetic resonance imaging environment: EFOMP recommendations ${ }^{\text {茠 }}$
}

\author{
J. Hand ${ }^{a, *}$, H. Bosmans ${ }^{b}$, C. Caruana ${ }^{\text {c }}$, S. Keevil ${ }^{a}$, D.G. Norris ${ }^{\text {d,e,f }}$, \\ R. Padovani ${ }^{g}$, O. Speck ${ }^{h}$
}

a Imaging Sciences \& Biomedical Engineering Division, King's College London, St Thomas' Hospital, London SE1 7EH, UK

${ }^{\mathrm{b}}$ Department of Radiology, Katholieke Universiteit Leuven, Leuven, Belgium

${ }^{c}$ Medical Physics Department, Faculty of Health Sciences, University of Malta, Msida, Malta

${ }^{\mathrm{d}}$ Donders Institute for Brain, Cognition and Behaviour, Radboud University Nijmegen, The Netherlands

e Erwin L Hahn Institute for MRI, Universität Duisburg-Essen, Germany

${ }^{f}$ MIRA Institute for Biomedical Technology and Technical Medicine, University of Twente, The Netherlands

${ }^{\mathrm{g}}$ Fisica Sanitaria, Az. Ospedaliero-Universitaria S. Maria della Misericordia, 33100 Udine, Italy

${ }^{\mathrm{h}}$ Department of Biomedical Magnetic Resonance, Otto-von-Guericke University, Magdeburg, Germany

Available online 9 January 2013

\section{KEYWORDS}

MRI safety;

MR Safety Officer;

MR Safety Expert
Abstract This European Federation of Organisations for Medical Physics (EFOMP) Policy Statement outlines the way in which a Safety Management System can be developed for MRI units. The Policy Statement can help eliminate or at least minimize accidents or incidents in the magnetic resonance environment and is recommended as a step towards harmonisation of safety of workers, patients, and the general public regarding the use of magnetic resonance imaging systems in diagnostic and interventional procedures.

(c) 2012 Associazione Italiana di Fisica Medica. Published by Elsevier Ltd. All rights reserved.

\footnotetext{
EFOMP Policy statement No.14 has been approved by EFOMP Council in Sofia (Bulgaria) on 20 October 2012. EFOMP Policy Statements are not subject to standard peer review as applied to other papers submitted to EJMP.

* Corresponding author. Imaging Sciences \& Biomedical Engineering Division, King's College London, St Thomas' Hospital, 1st Floor, South Wing, London SE1 7EH, UK. Tel.: +44 (0)20 7188 7118x53636; fax: +44 (0)20 71889154.

E-mail address: jeffrey.hand@kcl.ac.uk (J. Hand).
} 


\section{Introduction}

Magnetic Resonance Imaging (MRI) is the method of choice for imaging many parts of the body since it offers not only high resolution images of tissues but also metabolic and functional information non-invasively and without the risks associated with imaging techniques using ionizing radiations. MRI can also provide information to guide interventional procedures. Several professional groups contribute to the success and development of MRI, including radiologists, radiographers, physicists and engineers. MRI uses strong static magnetic fields, time varying magnetic fields produced by the gradient system, and radiofrequency electromagnetic fields. Potential hazards and safety issues arise from each of these components. Compliance with international $[1,2]$ and national [3,4] safety guidelines' and standards [5] has resulted in an excellent safety record for patients in view of the estimated more than 500 million MR examinations that have been performed worldwide. A Directive of the European Union that addresses occupational exposure to electromagnetic fields [6] is undergoing a revision and when implemented in its final form by member states is likely to impact on MRI practice, particularly regarding management of safety, user guidelines, and a harmonised training programme.

A recent survey of safety recommendations and regulations for MRI installations in European countries carried out by the European Federation of Organisations for Medical Physics (EFOMP) showed that there was a wide variation in procedures within Europe, ranging from compliance with national legislation that is more restrictive than international guidelines to reports of no regulation.

Medical Physics is an occupation recognised explicitly by the International Standard Classification of Occupations (ISCO-08) [7], and Medical Physicists are involved in the application of physics to challenges in healthcare. Although in the majority of countries Medical Physicists play a key role in ensuring safety within the MR environment, this is not true in all cases.

\section{Overview of potential hazards in MRI}

The greatest risk from the static magnetic field is the force exerted on ferromagnetic objects which accelerates them towards the scanner. Such projectiles have caused fatalities. Screening of patients for implanted ferromagnetic material is essential.

Movement of the body through the static magnetic field gradient can lead to transient sensory effects such as vertigo and nausea. In the cases of very high field systems, some people report a particular direction of apparent movement when standing close to the magnet even when stationary [8]. There are therefore implications for both patients and staff, particularly in and around high field systems.

The gradient system generates low frequency pulses ( $\sim 1 \mathrm{kHz}$ but with harmonics up to $\sim 10 \mathrm{kHz}$ ) which induce currents within body tissues that may result in peripheral nerve stimulation (PNS). PNS has no known long-term health consequences, but it can be unpleasant and, at sufficiently high levels of exposure, even painful. The pulsed currents in the gradient coils also interact with the static magnetic field and give rise to Lorentz forces which expand and compress the coil mountings. These vibrations are transmitted through other structures in the scanner to the environment, generating a loud noise. The acoustic levels are sufficiently high that hearing protection is advised to avoid discomfort and possible shifts in hearing thresholds.

The radiofrequency magnetic field and its associated electric field deposit energy within the tissues which may lead to tissue heating. This depends also on the type of pulse sequence and specific pulse sequence parameters used. In some situations, for example when coil leads are placed on the skin or conducting loops are formed by the patient's arms and hands in contact with their trunk, this can be excessive and lead to local burning of tissue. Local heating may also occur around implanted conducting structures such as guide wires and some prostheses.

\section{MRI safety management}

It is essential to ensure the safety of staff, patients, volunteers, and visitors within the MR environment. EFOMP suggests that a 2-level approach to the management of MRI Safety which distinguishes between the roles of the person responsible for MR safety on a day-to-day basis and those of an expert level professional with a higher degree of scientific and technical expertise in MRI is adopted. This mirrors the case of ionising radiation where the new revised EC Basic Safety Standards directive [9] mandates the existence of two levels, an Officer level and an Expert level.

\section{The MR Safety Officer}

The first of these, the MR Safety Officer (MRSO), must

- be competent to assess and manage dangers caused by the MR equipment

- be responsible for monitoring the measures taken to protect against such dangers

- ensure that appropriate measures for minimizing risks to health that arise from the use of the MR equipment are implemented and monitored

Required knowledge and competence include the general principles of good safety management, the need to develop, document and introduce safe working procedures, and possession of adequate technical understanding and management skills to carry out the administrative responsibility delegated, and these should be acquired through a formal, recorded training.

This level of safety provision is included, for example, in the Austrian standard ÖNORM S 1125-1 [10] (the MRSO) and by the MR Responsible Person (MRRP) recommended by the UK's MHRA [3]. The role of MRSO may be carried out by suitably qualified personnel having recorded training such as Medical Physicists, Radiographers, Radiologists, etc.

\section{The MR Safety Expert}

The second role - the MR Safety Expert (MRSE) - requires the skill, knowledge and competence to provide high level advice on the engineering, scientific and administrative 
aspects of the safe clinical use of MR devices. In particular the MRSE should be responsible for:

- the development and continuing evaluation of a safety framework for the MR environment

- the development of local rules and procedures to ensure the safe use of MR equipment

- advice regarding non-routine MR procedures for individual patients and specific patient groups (including competence regarding safety related to implanted devices and tattoos).

The purchasing of MRI equipment should be undertaken by a consultation group consisting of a wide range of personnel. In this context the expertise of the MRSE is an important source of:

- safety advice regarding the selection and procurement of $M R$ and related equipment

- advice on the design for accommodation and facilities for MR equipment.

The MRSE also has expertise in acceptance testing prior to the first clinical use of the equipment, in regular performance testing, and testing following any major maintenance procedure.

The role of MRSE requires a higher level of knowledge, skill, and competence than the MRSO, in line with, for example, recommendations by the UK MHRA [3] (the MR Safety Adviser) and requirements for the MRSE in Italy [4] who is responsible for the safety of staff, for the safe installation of equipment, and for a quality control programme regarding performance of the equipment. However, currently within Europe there is no consensus regarding the skills, knowledge and competence required of the person responsible for MR safety. For example, in Germany there are no requirements for this person to be from a specific profession or to have any accredited training.

EFOMP recommends that appointees should hold relevant scientific qualifications, including sufficient $M R$ physics, which should be at least at European Qualifications Framework (EQF) level 7 (with 1 or more additional years of relevant hands on experience).

EFOMP also recommends that accreditation of MRSEs should be provided by a competent authority and that a periodic review of appointees at 5 year intervals will facilitate harmonization of training and experience of MRSEs. To maintain accreditation EFOMP would expect the MRSE to demonstrate continuing professional development (CPD) including further advanced training to ensure that they provide effective and safe practice and advice based on current best evidence or own research when the current evidence is insufficient. Such training should be aimed at achieving EQF level 8.

EFOMP realises that although such high level expertise is usually available in large public sector institutions, it may not be available in every MR facility. When advice at this level is required in these cases, the expertise can be sourced from a large institution or an independent sector provider. In the cases of public sector institutions with a number of geographically dispersed MR installations and sites and/or mobiles operated by an independent sector provider, a single MRSE could provide the required expertise.
Although in principle the required skills, knowledge and competence to advise on the safety of MR devices at the level required of the MRSE is not exclusively the domain of Medical Physicists, the UK's MHRA [3] recommends that the high level expert (the MR Safety Adviser) should be a physicist with expertise in MRI (and in the clinical environment should normally be a Clinical Scientist [11] (a protected title and registered profession in the UK)).

Some Medical Physicists work in MR within conventional imaging departments where a background in medical applications of ionizing radiation is also needed. In these situations a single medical physicist may possess expertise in both areas, or the roles may be discharged by different individuals. There is a growing role of MRI outside conventional imaging departments such as in MRI specific neurology, surgery, and cardiology departments where medical physics expertise in ionizing radiation may not be needed.

One route to accomplish the education and training appropriate for an MRSE is via the Medical Physics Expert (MPE) [9] specialised in Diagnostic and Interventional Radiology and with extensive experience in MRI. However, other routes to acquiring the necessary skills, knowledge and competence are equally valid.

Most Medical Physicists with the knowledge, skills and competence required of the MRSE will also contribute to the wider scientific support to a clinical MR department thereby adding further value to the appointment.

\section{Recommendations}

The Council of EFOMP recommends:

- a 2-level approach to management of safety within MR units through the MR Safety Officer (MRSO) and the MR Safety Expert (MRSE).

- that the MRSO should undergo formal recorded training to achieve the appropriate skill, knowledge and competence to be responsible for day-to-day safety within the MR unit.

- that the MRSE should have knowledge, skills and competence and qualifications outlined above

- that accreditation of MRSEs should be provided by a competent authority and reviewed periodically at 5 year intervals.

- that the professional appointed as the Magnetic Resonance Safety Expert (MRSE) should preferably be a Medical Physicist as defined in the International Standard Classification of Occupations (ISCO-08) under group 2111.

These recommendations are addressed to organisations in Europe with vested interest in the safety of workers, patients, and the general public regarding the use of magnetic resonance imaging systems in diagnostic and interventional procedures.

\section{Acknowledgements}

The authors acknowledge all EFOMP's Officers who have contributed to the final version of this document with comments and suggestions. 


\section{References}

[1] International Commission on Non-lonizing Radiation Protection (ICNIRP). Medical magnetic resonance (MR) procedures: protection of patients. Health Phys 2004;87:197-216.

[2] International Commission on Non-lonizing Radiation Protection (ICNIRP). Amendment to the ICNIRP 'Statement on medical magnetic resonance (MR) procedures: protection of patients'. Health Phys 2009;97:259-61.

[3] Medical and Healthcare products Regulatory Agency (MHRA). Safety guidelines for magnetic resonance imaging equipment in clinical use. DB2007(03). London: MHRA; 2008.

[4] Italian Ministry of Health Decree of 2nd August 1991 and Decree of the President of the Republic no. 542 of the 8th August 1994.

[5] International Electrotechnical Commission (IEC). Medical electrical equipment: part 2-33. Particular requirements for the safety of magnetic resonance equipment for medical diagnosis. IEC 60601-2-33 ed 3. Geneva: IEC; 2010.

[6] Directive 2004/40/EC of the European Parliament and of the Council of 29 April 2004 on the minimum health and safety requirements regarding the exposure of workers to the risks arising from physical agents (electromagnetic fields) (18th individual Directive within the meaning of Article 16(1) of Directive 89/391/EEC). OJ L 30.4.2004;159:1-26.

[7] International Standard Classification of Occupations (ISCO). Geneva: International Labour Organisation; 2008. http:// www.ilo.org/public/english/bureau/stat/isco/isco08/index. htm.

[8] Glover PM, Cavin I, Qian W, Bowtell R, Gowland PA. Magneticfield-induced vertigo: a theoretical and experimental investigation. Bioelectromagnetics 2007;28:349-61.

[9] Proposal for a Council Directive laying down basic safety standards for protection against the dangers arising from exposure to ionising radiation, http://ec.europa.eu/energy/ nuclear/radiation_protection/doc/com_2011_0593.pdf; 2011.

[10] Austrian Standard (ÖNORM) S 1125-1. Sicherheitsbeauftragter für Magnetresonanz-Tomographiegeräte in der medizinischen Diagnostik Teil 1: Verantwortlichkeiten und Zuständigkeiten; 2009.

[11] http://www.hcpc-uk.org/aboutregistration/professions/index. asp?id $=4 \#$ profDetails. 